\title{
Towards a better understanding of the full impact of the left digit effect on individual trading behaviour: Unearthing a trading profit effect
}

\begin{abstract}
Investors' perceptions of price have been shown to be disproportionately affected by the leftmost digit(s). However, a similar left digit effect $(L D E)$ in relation to another important determinant of investors' behaviour (i.e. trading profit) has not been explored. We examine over 7,314,570 million trades made by 25,766 individuals and find a LDE in profit that is 1.71 times stronger than that related to closing price; suggesting that individuals focus more on left digits in profit than price when deciding when to close a trade. In addition, we observe a positive synergistic relationship between the LDE related to profit and price, suggesting that its total influence may result in losses of billions of dollars per financial year for investors. We suggest that these results make a strong case for educating investors against this bias.
\end{abstract}

Keywords: Left Digit Effect, individual trading behaviour, heuristics, round numbers in profit

\section{Introduction}

The left digit effect (LDE), whereby individuals' choices are disproportionately affected by the left-most digit(s), has been found in a wide range of fields from goal setting to buying and selling assets (Sopranzetti and Datar 2002; Sonnemans 2006; Pope and Simonsohn 2011; Allen et al 2016). Retailers, for example, take advantage of the LDE by pricing just under round numbers; individuals prone to the bias perceiving $£ 1.99$ as markedly lower than $£ 2.00$. Bhattacharya et al (2012) claim that investors subject to the LDE, are more likely to trade around round number prices and that this leads to negative abnormal returns of around $\$ 1$ billion per annum on the New York Stock Exchange (NYSE). The significant economic impact on investors of the LDE related to prices, motivated us to explore if this effect also relates to another, possibly more important metric affecting investment decisions, namely an investor's current profit. It is surprising, given that the foremost aim of investment is securing profit, that 
the impact of LDE related to profit has not to date been examined. The profit (loss) per trade on an asset/portfolio has been shown to be an important determinant of the well-researched cognitive bias, the disposition effect (Shefrin and Statman 1985). Similarly, profit and loss play important roles in another cognitive bias, mental accounting (Thaler 1985), traders tending to integrate losses and segregate profit, leading them to make risky investments if they are making losses (Lim 2004). It has been shown that automated self-discipline mechanisms such as limit/stop orders can help reduce some cognitive biases, such as the disposition effect (Fischbacher et al, 2017; Richards et al, 2017). However, orders using these mechanisms continue to cluster around round numbers, suggesting they have not helped in overcoming the LDE (Aşçığlu et al, 2007; Bourghelle and Cellier 2009; Kuo et al, 2014). This is a further motivation for better understanding the full extent and economic impact of the LDE, since this may help to stimulate the search for ways of reducing the bias.

This paper highlights the importance of profit as a metric influencing the LDE and the necessity to examine a range of metrics that could influence the LDE, if its true economic impact is to be discerned. We examined 7,314,570 spread trades made from 2004 to 2013 by 25,766 individual traders to highlight the presence, salience and interaction of the LDE in profit and closing price. We find that there is a LDE related to both profit and price and that profit has a bigger effect; suggesting that the impact of a metric on the LDE is dependent on the importance that investors ascribe to that metric. In addition, we find that there is a positive and synergistic effect of profit and price on the LDE and that the full economic impact of the LDE has been significantly under-estimated by studies that have focussed on the impact of price alone. In fact, we find that the LDE in profit to be 1.71 times larger than that related to closing price and the synergistic effect related to both profit and closing price magnifies investors' net negative returns due to the LDE to many billions of dollars per financial year. These results suggest that the LDE may be having a very significant economic impact and heightens the need 
to make all classes of investor aware of this bias. This is particularly true with the advent of high frequency automated trading algorithms that can be programmed by sophisticated and unsophisticated traders alike; potentially exacerbating the impact of the LDE.

The remainder of the paper proceeds as follows: In section 2, we highlight relevant literature related to the LDE. In addition, we explore literature which suggests that profit is the primary metric in financial decision-making and we examine Information Integration Theory (IIT), which explains how individuals' cognitively process two pieces of relevant information, possibly resulting in a synergistic outcome. These literatures reviews are used motivate the hypotheses to be tested. In Section 3, we outline the data and methodology employed to test the hypotheses. We present our results in Section 4 and demonstrate that these provide support for the hypotheses. In Section 5, we discuss our findings in the light of previous literature. Finally, in the conclusion (Section 6) we summarise our findings and suggest that interventions are needed to aid traders in their decision making processes.

\section{Literature Context and Hypothesis Development}

\subsection{The Left Digit Effect}

The LDE in the field of consumer theory is also known as odd pricing and nine ending pricing, whereby, consumers are shown to react differently to price points ending in a nine compared with zero (Bray and Harris 2006; Manning and Sprott 2009, Levy et al, 2016). It is argued that this arises from a combination of the level and image effects (Stiving and Winer 1997; Thomas and Morwitz 2005). The level effect involves individuals subconsciously rounding down to the next integer (e.g., $£ 2.99$ perceived as $£ 2.00$ ), because digits are processed on an analogue mental scale from left to right; the leftmost digit carrying more value than the subsequent digits. The image effect involves consumers associating prices ending in a nine with discounts (Schindler and Kibarian 2001). Consequently, £3.99 is considered a far more desirable price than $£ 4.00$. Consumers, when recalling the price paid, tend to remember the leftmost digits, suggesting they impart less significance to the other digits (the memory effect: Coulter 2001). 
A number of factors determine when the LDE is strongest. In particular, the distance effect, suggests that the LDE is stronger in comparing numbers that are closer to each other (e.g., between $£ 1.99$ and $£ 2.00$, rather between $£ 1.99$ and $£ 3.00)$ (Thomas and Morwitz 2004). The size congruency effect suggests that if smaller numbers appear on the left then this decreases the magnitude of the LDE (e.g., $£ 1.99$ vs $£ 2.00$ would result in a weaker LDE than $£ 2.00$ vs $£ 1.99$ ). In addition, if the prices to be compared are displayed vertically, the LDE is diminished (Lin and Wang 2011). Kuo et al (2014) suggest that the LDE in prices increases among less sophisticated retail investors and that the LDE weakens due to a learning effect (based on number of trades and trading duration). Furthermore, Fraser-Mackenzie et al (2015) suggest that the LDE is stronger when a trade is in loss

\subsection{LDE in Financial markets: Profit vs. Price}

Clearly, in financial markets the difference between the price at which an asset is bought and sold is the profit (or loss). Therefore, both the price and profit metrics are interlinked and affect each other.

Donaldson and Kim (1993) looked at price barriers on the Dow Jones Industrial Average as investor psychological reference points. They found that these price barriers, which form, support or resistance levels, aided the rise and fall of the index. The price barriers were in multiples of the round number 100 (for example 2900, 3000 and 3100 etc.). Sonnemans (2006) also found evidence of price barriers on individual stocks on the Dutch stock market, whereby investor sentiment resulted in clustering on round numbers ending with digits zero and five. Doucouliagos (2005) found price barriers on the Australian stock exchange. This study, along with that of Pope and Simonsohn (2011) suggest that these price barriers, which are formed on round numbers in powers of 10, arise due to the impact of the decimal system on cognitive reasoning, thereby forming mental reference points. Individuals trading exclusively around round number price barriers in price, suffer monetary loss. According to 
Bhattarcharya et al (2012), investors who exclusively trade just below round numbers have been shown to make negative abnormal returns of around \$1 billion on the NYSE in one financial year (in simulated trading).

Similarly Johnson et al (2007) showed that net profit when trading in nine-ending stock prices is significantly less than that associated with trading stocks just above a round number. However, these studies do not suggest that LDE in profit may directly impact trading decisions.

While profit and price are interlinked, profit is a more important metric in influencing decision making in many settings. A variety of industries work backwards in setting a market price by reducing costs. Known as target costing, this managerial practice involves pricing a product based on a predetermined profit margin (Ansari et al, 2006). Research has found that these margins, expressed as percentages, tend to be in hurdles of round numbers $(10 \%, 20 \%$ etc.) (Carr and Nanni 2009). This implies that predetermined profits at an organisational level are prone to LDE and highlights the importance of profit in the decision-making process.

Behavioural biases associated with profit (as well as price) secured by an individual can determine when a trade is opened and/or closed. A prominent cognitive bias, which utilises profit as a decision-making metric, is the disposition effect. It involves selling profitable investments too quickly and holding onto loss making investments too long (Kahneman and Tversky 1979; Shefrin and Statman 1985). The disposition effect has been observed in many markets and its widespread application suggests that the profit level achieved figures strongly (perhaps more so than price) in the decision to close or keep open an individual trade.

The level of profit achieved is also a key ingredient in a further psychological concept linked to how individuals prioritise decisions, namely mental accounting. This states that human beings divide their money into mental accounts or independent silos and their decisions to spend money from these accounts are influenced by prior spending linked to those accounts. A rational thought process would suggest that money is interchangeable between accounts and 
independent of past outcomes (Thaler 1985). However, professional daily stock market traders appear to open and close mental accounts every day, resulting in them tending to trade excessively in the afternoon to claw back losses before the close of trading that day (Garvey et al, 2007; Lim 2004). This arises because risk averse traders want their daily mental account to be positive. The widespread use of mental accounting suggests prior profits and losses are important determinants of an individual trader's decision to open and close a trade.

As indicated above, individuals prefer round numbers for the ease of recall and for the jump in perceived magnitude that occurs when increasing from a number ending in nine. This bolsters cognitive convenience and utility. Given that investors have a choice of determining when to close a trade, we believe that there will be a higher probability of them closing a trade when the realised profit is a round number (e.g., with a rightmost digit 0 or 5). This aspect of the LDE has not been explored in previous studies, so, we test the following hypothesis:

H1: Individuals exhibit a left digit bias by closing individual trades, which secure roundnumber profits.

We believe that the salience of the LDE is likely to be dependent on the importance ascribed to a metric by the individual. Thus, we compare the strength of the LDE in two important simultaneously occurring metrics - profit and closing price. The foremost aim of most investors is to make a profit. Equally, research related to the disposition effect and mental accounting, discussed above, also points to the greater salience of profit (cf. price) in the decision of when to conclude a transaction. Consequently, we test the following hypothesis: H2: When closing single trades, individuals are more influenced by left digit bias associated with the profit secured than that associated with the closing price.

\subsection{Preferring a Rounder Digit}

Bhattacharya et al (2012) documented round-number bias in stock prices alone. They found that price clustering has a clear hierarchy: investors preferring "rounder numbers" from the 
'most round' zero ending digit to the least round in multiples of five (the "threshold figure" effect). For example, investors would prefer the following numbers in descending order when buying and selling: $.00, .05, .025$, etc. This view is to some extent supported by the fact that that limit and stop orders (which offer investors more choice with predetermined prices to buy/sell) also cluster around round numbers, with a much higher preferences for zero ending prices (Aşç1oğlu et al, 2007; Bourghelle and Cellier 2009; Kuo et al, 2014).

This preference for rounder numbers is not limited to financial trading. A study by Lynn et al (2013) found that consumers in pay-what-you-want situations, such as tipping waiters or pumping gasoline, were subject to the LDE. They preferred to pay in rounder figures ending in zero (even cf. figures ending in five), with the rightmost digits 00 being the most preferred option. One reason for this preference may be that zero is a larger cognitive reference point or mental shortcut, making recall of price paid and cognitive processing easier.

We aim to explore if the preference for rounder digits exists in terms of both closing prices and profit. If this were the case, it might point to a universal set of properties for the LDE that are transferable across metrics. As profit allows investors more freedom to determine which digit to utilise, it is important to document this increased preference, as this might partially explain any higher salience of the LDE in profit compared to closing price; i.e. suggesting that accessibility to rounder digits is one of the reasons for increased salience of the LDE in any numerical metric. To explore this we test the following hypothesis:

H3: While closing a single trade, investors subject to left digit bias will display a preference for rounder numbers in both closing price and profit.

\subsection{LDE in Profit and Price: The Combined Effect}

The combined cognitive biases hypothesis states that, rather than working in isolation, behavioural biases act together. For example, it has been shown that several biases in a wellresearched psychological phenomena such as depression tend to have a greater overall impact 
acting together (Everaert et al, 2012; Hirsch et al, 2006). Chen et al (2007) highlights that all types of investors suffer from multiple cognitive biases, and these can occur simultaneously. This may be particularly true for retail investors. Furthermore, Chu et al (2012) suggest that overconfidence (trading excessively and unnecessarily) results in a stronger disposition effect, thus highlighting a positive relation between the two biases. Chou and Wang (2011) demonstrate that, subject to positive prior returns, both overconfidence and the disposition effect act in unison and are responsible for a surge in trading volume. This suggests that cognitive biases can have a positive relationship, amplifying the overall impact on decisions. However, the application of overconfidence and the disposition effect are not based on a common visual numerical stimulus; unlike the LDE. While trading online, regardless of the electronic device used, traders view closing price and profit simultaneously. Both these metrics are pieces of information. Anderson (1971) suggests, via the framework of the Information Integration Theory (IIT), that individuals combine different pieces of information to help them form judgements and decisions. Each piece of information has a value (positive or negative) and weight. Consequently, any new information is added, multiplied or averaged to form a new opinion that depends on the circumstance and the individual. We expect those traders who are prone to the LDE in profit/price to view a round-number closing price/profit as a positive piece of information inducing them to close a trade. Thus, a round number profit and a round number closing price occurring at the same time is likely to accelerate the decision to close the trade.

The IIT framework aids in explaining when different sources of information might be integrated in a manner that produce a combined impact greater than that attributable to adding the impacts of the two pieces of information together. Kim et al (2010) use this to suggest that different advertising and good publicity information about a product may be positively integrated by consumers to create a synergistic effect, resulting in a spike in sales. This more than additive positive integration, which relies on reaffirming existing beliefs, also extends to 
complimentary brand alliances, resulting in higher sales for all the firms involved (Rodrigue and Biswas 2004).

We suspect that different traders may be prone to different degrees of the LDE in both profit and closing price. However, when both profit and closing price are round numbers, they will form two consistent pieces of belief-reaffirming information. The IIT framework then suggests these pieces of information will be positively integrated and will have a synergistic influence on the decision to close a trade. Consequently, we believe that the simultaneous application of the LDE associated with both profit and closing price will substantially increase the possibility of an investor closing a trade. Consequently, we test following hypothesis:

H4: The combined influence of LDEs related to the profit secured and the closing price of a trade will be synergistic in nature.

\section{Methodology}

There are around half a million active retail spread traders in Britain, and this number is rising rapidly. Traders do not own the underlying asset, but rather speculate on its rise (long) or fall (short). We secured our data from a leading UK based spread trading provider (brokerage and trading platform). Traders can register to trade on their website (or online channel) allowing them to buy/sell several financial instruments based on price quotes listed.

As the traders do not own the underlying asset and are speculating on future direction to make a profit, spread trading providers tend to mimic the underlying prices of assets (with the addition of a fixed brokerage fee) at which they trade on an underlying exchange (such as the FTSE). Most spread trading providers (including the one from which we obtained the data) have a fixed brokerage amount (which can vary by asset), which traders are made aware of before they can start trading. The prices quoted to traders are then the underlying market price of the asset plus the brokerage. For example, if the FTSE was trading in the underlying market at 7400, and the brokerage charged 1 point, then a trader wishing to 'buy/sell' the FTSE would 
not make any profit until the underlying market price exceeded/was less than, 7401/7399. Given that this brokerage fee is fixed, it would not be legally feasible to manipulate the prices quotes to be round numbers. Equally, given that profit is a function of the difference between opening and closing price, if the prices quoted are not manipulated it would be difficult to manipulate profit to be a round number.

Therefore, the trader is buying/selling from the spread trading provider who acts as a market maker and guarantor of transactions. In addition, these firms buy/sell the actual underlying asset as a hedge to protect against severe losses. In this way, they can influencing the primary market.

We employed data from spread trading markets as they have several advantages in measuring the LDE in profit and closing price. In particular, as the provider is located in the UK, the greatest advantage is that profits from spread trading are tax-free. This ensures that decisions of traders are not influenced by tax considerations (e.g., seasonal factors may appear where traders seek to reduce capital gains tax: e.g., Odean (1998) and Dhar and Zhou (2006)).

As the traders do not own the underlying asset, the probability of a buy/sell order being unfulfilled is limited, such as rare times of technical difficulty. Therefore, our data includes almost the entirety of the decisions made by an individual trader with the spread trading company.

$\mathrm{n}$ addition, since all the trading we observe is electronic in nature, during the lifetime of every trade a trader can view net unrealised profit alongside price on the screen. The costs of credit or leverage is already subtracted from the net profit visible to the trader. In addition, all prices quoted contain the transaction costs (i.e. cost of brokerage). If the profit on display was gross profit, as is the case with traditional retail stock broking, traders may be inclined to try to calculate net profit by mentally deducting transaction costs while a trade is open. With 
rapidly changing prices, this delay may cause them to overshoot or undershoot a round number cognitive reference point, thereby reducing the measurable impact of the LDE in profit.

The raw data consisted of $8,441,805$ trades made by 28,211 traders in four of the most popular markets from $8^{\text {th }}$ November 2004 to $31^{\text {st }}$ March 2013: the DAX 30 and FTSE 100 rolling indices and the currency pairs EUR/USD and GBP/USD. Thus, we are able to focus on spread trades associated with the FTSE and DAX, two of the largest stock markets by market capitalisation and volume in Europe. They act as a metric to measure the performance of the entire stock exchange. Furthermore, because the US dollar is the world's reserve currency, the currency pairs in our sample $(£ / \$$ and $€ / \$)$ represent some of the most traded in the world. The significance of the markets we employ gives us confidence that our results are likely to be applicable to a wide cross-section of markets.

\subsection{Data}

In our data set, for every trade, the opening and closing price, trade direction (long or short), pip size and pip value (stake size) are electronically recorded in an automated manner. To calculate profit, opening and closing nominal asset prices are divided by a pip size and the this, due to the differing nature of markets, varies (e.g., indices generally have a pip size of 1 while currency pairs have a pip size of 0.0001). We employ these standardised, rather than raw, prices (i.e. raw prices divided by the pip value) because the indices and forex assets traded are associated with different absolute values. For example, with no cost of leverage, a trade on the GBP/USD currency pair may have an opening value of $£ 1.6790$ and a closing value of $£ 1.6795$. Both values are divided by the pip size of $£ 0.0001$, giving us a standardised price of 16,790 and 16,795. The difference between the opening and closing standardised price (5) is multiplied by the stake on the trade (say $£ 1$ ) to give a profit of $£ 5$. 


\subsection{Excluded Trades}

Limit orders involve investors making pre-determined trading decisions and an investor does not have to be actively trading for a limit order to be executed. With round-number prices being popular due to the LDE, limit orders may not be filled due to buy/sell imbalances. According to Bhattacharya et al (2012), due to the "cluster undercutting effect", some investors place limit orders on digits just above or below round numbers to ensure they are fulfilled. Equally, Linnainmaa's (2010) study of limit order patterns suggested that limit orders do not convey the true intentions of investors due to the artificial mechanisms involved. This suggests that even though many traders would prefer round-number prices their preferences cannot be identified in limit order prices, thereby leading to an under-estimation of the LDE. Consequently, we did not examine any limit order transactions (768,779 trades). Rather, we limited our analysis to market transactions, which involved investors actively opening or closing a trade at the prevailing market price.

In order to further explore the impact of LDE in relation to profit, it would be useful in future studies to examine if traders attempt to lock in round number profits when executing limit orders. Unfortunately, the sample used in this study did not contain sufficient limit orders for this analysis to be conducted.

As indicted above, in spread trading, asset prices are converted into a standardised form by dividing them by their pip size. This results in all asset classes having prices, which are either four or five digits in length. Fraser-Mackenzie et al (2015) investigated LDE in prices in spread trading and found that investors only focus on the first three digits from the right. This is because the fourth and fifth digits seldom change. In order to prevent the results being biased by unusual trading pattern on the rare occasions when the fourth or fifth digit changed, we excluded the 16,306 trades, where this was the case. 
Despite being a round number, the realised returns figure of 0.0 is neither a profit nor a loss. Furthermore, due to cognitive biases associated with mental accounting (e.g., Thaler and Johnson 1990), traders are often particularly attracted to close trades which have been in loss as soon as the position returns to a break-even point, as Thaler and Johnson (1990) claim that investors hate realising losses in their mental accounts. Similarly, the house-money effect would suggest that if a trade immediately upon opening is profitable, the trader would perceive themselves to be in surplus in their mental account. Thaler and Johnson (1990) suggest that these trades are left open regardless of risk, as the profit is not deemed to be the investors' but rather the house's (broker's) money. To avoid our measurement of the degree of the LDE being combined with the effect of the break-even and house money effects we excluded trades with returns of 0.0 in realised profit (256,861 trades). As a result of our data pre-processing, this left $7,314,570$ individual trades made by 25,766 traders for the analysis outlined in Section 3.4.

\subsection{Descriptive Statistics}

The median $(£ 2.30)$ and mean $(-£ 3.62)$ values for realised profit on a single trade appear to indicate that realised profits in general do not deviate far from $£ 0$. However, the difference between the maximum $(£ 113,956.00)$ and minimum $(-£ 189,810.00)$ realised profit figure for a single trade indicates that there is a wide variation in the profit secured on a single trade, and this is confirmed by the high value of the standard deviation (£314.96). The data displayed a high positive kurtosis $(35,091.29)$ implying that the realised profit values are clustered around the mean and median values. We observe a negative skewness (-72.24) in realised profits, implying that more trades are closed in profit than in loss. This is also confirmed by the positive median value of realised profit we observe. In total, $44.24 \%, 46.00 \%, 9.19 \%$ and $0.57 \%$ of trades secured a realised profit (cf. loss) of one, two, three and four or more digits, respectively. Thus, $90.24 \%$ of trades had a realised profit of two digits or less 
In summary, the key finding in relation to our study from the descriptive statistics is, despite a considerably large standard deviation, the majority of individual trades involve small amounts of profit (cf. loss) ranging between $£ 99$ and $-£ 99$. These values are similar to prices in studies on LDE associated with everyday items (Bray and Harris 2006; Levy et al, 2011).

\subsection{Data Analysis}

Closing price and profit are two simultaneously occurring metrics that can influence an individual's decision to close a trade and left digit bias is demonstrated by a greater frequency of occurrence of round numbers in price and profit. Consequently, we employ two categorical variables, the rightmost digits of profit and closing price. Each variable can take ten values between 0 and 9 for any individual trade.

A significant minority of traders had made only a handful of trades (e.g., 1,515 traders (5.88\%) had made a single trade and 6,738 traders (26.15\%) had made ten trades or less). This suggested our sample consisted of a large number of traders with minimal trades and a small number of traders with large number of trades. We therefore decided to aggregate data, as making inferences via parametric regression analysis might potentially be biased given the lack of sufficient trades by a large segment of traders. We therefore examined the frequency of occurrence of closing prices and profits on round numbers across all trades. However, to ensure that our results were not skewed by the most frequent traders, we examined whether similar results were obtained for the bottom quartile of traders (in terms of number of trades) and the top $10 \%$ and $1 \%$ of most frequent traders.

Kateri (2014) argues that a contingency or cross-tabulation table is ideal for analysing the relationship between two categorical variables such as those examined here. In particular, it has the advantage of aggregating data and dividing it into visually easy and precise categorical combinations, highlighting all possible interactions, making comparisons easier and enabling examination of relationships within the data that might not be readily apparent. 
Furthermore, Camili and Shepard (1994) point to the fact that its use on non-parametric inferences ensures that the analysis is not constrained by prior assumptions on variables distributions. These advantages have led to the use of contingency tables for a range of financial market studies, including persistence analysis in ranking the performance of portfolios in investment and mutual funds (Casarin et al, 2005; Cortez et al, 1999; Lean et al, 2015). This approach is particularly helpful in testing our second hypotheses related to the relative strength of the LDE in closing price and profit.

The price at which a trader decides to close a trade will determine their realised profit. The price and the profit could, therefore, simultaneously, affect a trader's decision of when to close a trade. The rightmost digit of profit and of closing price can take up to ten values (0-9) and the frequency of occurrence of round numbers in the rightmost digits for these metrics will indicate whether profit, closing price or both are affected by the LDE. For example, if there is no preference for round numbers in realised profit (or closing price), $10 \%$ of trades will be closed at a profit (closing price) whose rightmost digit ends in $0,10 \%$ of trades will be closed at a profit (closing price) whose rightmost digit ends in 5, and each of the other digits between 1 and 9 will occur approximately $10 \%$ of the time. Consequently, we constructed a $2 \times 9$ contingency table (Table 1) combining the data of the rightmost digit in closing price and profit from each trade. We examined the number of trades where the rightmost digit of closing price was either 0,5 or Other (i.e. digits other than 0 and 5). The expected frequency of occurrence of each pair of the rightmost digit of profit and closing price is the expected percentage frequency of the rightmost digit of profit multiplied by expected percentage frequency of the rightmost digit of closing price. For example, the profit/price rightmost digit combination 00 has an expected frequency of $1 \%$ (i.e. $10 \%$ x $10 \%$ ). 
Table 1. Contingency Table of the Expected Values of Rightmost digit Profit and Closing Price Combinations

\begin{tabular}{ccc}
\hline $\begin{array}{c}\text { Rightmost } \\
\text { Digit } \\
\text { Profit / Price }\end{array}$ & $\begin{array}{c}\text { Expected } \\
\text { Frequency }\end{array}$ & $\begin{array}{c}\text { Expected } \\
\%\end{array}$ \\
\hline O/O & $73,145.7$ & $1 \%$ \\
O/5 & $73,145.7$ & $1 \%$ \\
O/Other & 585,180 & $8 \%$ \\
$5 / 0$ & $73,145.7$ & $1 \%$ \\
$5 / 5$ & $73,145.7$ & $1 \%$ \\
$5 /$ Other & 585,180 & $8 \%$ \\
Other/O & 585,180 & $8 \%$ \\
Other/5 & 585,180 & $8 \%$ \\
Other/Other & $4,681,440$ & $64 \%$ \\
\hline
\end{tabular}

A Pearson's goodness of fit $\mathrm{chi}^{2}$ test was conducted to check whether the observed distribution of the profit/price rightmost digit combinations was different from the expected values. The null hypothesis is that there is no difference between the observed and expected values, implying no LDE. If this null hypothesis is rejected, we implement post hoc tests.

In particular, we conduct two-sided proportions tests that make use of underlying frequency as a proportion of total sample size when comparing two populations. Making use of all our trades (total sample), we explore if the difference between the proportion of expected and observed frequencies of a single profit/price rightmost digit combination is significant. This extends to comparing the difference between two profit/price rightmost digit combinations. The null hypothesis suggests that there is no difference in the frequencies being compared. As we expect preference for round numbers, profit/price rightmost digit combinations involving round digits should be different and overrepresented. Therefore, unless the null hypothesis of our proportions test is rejected, there is no left digit bias.

Our first Hypothesis, which posits that there is a LDE in profit, is tested by examining the rightmost digit profit/closing price combinations 0/Other and 5/Other in Table 1. These 
combinations involve the rightmost digit of profit being a round number alongside the rightmost digit of closing price not being a round number. This was to avoid the LDE in price (as has been found in previous studies), influencing $L D E$ in profit. In other words, if LDE in profit is present, this should be displayed in these combinations. Consequently, we conducted a two-sided proportions test on the expected and observed frequencies of the 0/Other and 5/Other combinations, to test whether these were underrepresented or overrepresented. Thereby providing evidence to accept or reject $H 1$.

The second Hypothesis posits that there is more LDE in profit than in closing price. To test this hypothesis, we conducted a two-sided proportions test on the expected and observed frequencies of two rightmost digit profit/price combinations sets 0/Other vs. Other/0 and 5/Other vs. Other $/ 5$, which appear in Table 1. If the combination 0/Other is over-represented (as suggested by the proportion test) compared to the combination Other/0, it suggests that the LDE in profit is more salient than the LDE in closing price for the digit 0 . Similarly, if the combination 5/Other is over-represented (as suggested by the proportion test) compared to the combination Other/5, it suggests that the LDE in profit is more salient than the LDE in closing price for the digit 5

H3 posits that investors demonstrate a preference for the 'rounder' digit 0 over the digit 5. To test this hypothesis, we therefore conducted two-sided proportions tests on rightmost round digit combinations of profits and closing prices which appear in Table 1. If a proportion test between the rightmost digit, profit/price combinations $0 / 0$ and $0 / 5$ (in which the rightmost digit 0 in profit was constant) suggests the rightmost digit 0 in closing price is relatively overrepresented, this suggests that investors preferred the rounder rightmost digit 0 in closing price (over the digit 5). Similarly, if a proportions test between the combinations $0 / 0$ and 5/0 (in which the digit 0 in closing price was constant), suggested that the 0 in profit was relatively 
overrepresented, it would imply that investors preferred the rightmost digit 0 in profit over digit 5 .

$H 4$ posits that there is a synergistic interaction between LDE in profit and closing price. Subject to there being evidence for $\mathrm{H3}$, we focus only on the rightmost digit 0 in profit and closing price to test H4 (with digit 5 being included in the category 'Other'). Therefore, our 2 $x 9$ contingency table is compressed into a $2 \times 4$ contingency table, with combinations 0/0, O/Other, Other/O and Other/Other. We first calculate the LDE in profit and closing price if these cognitive biases act independently. The proportions of trades associated with combinations 0/Other and Other/O demonstrate, respectively, LDE in profit and in closing price. Multiplying the proportion of trades of the combinations Other/0 and 0/Other provides an expected proportion (which can be translated into expected frequency) of trades if LDE in profit and closing price act independently. The expected frequency is compared (via a proportions test) to the observed number of trades of the combination 0/0. If the proportion test suggests that the observed frequency 0/0 combination is greater than the independent LDE expected frequency, it supports $H 4$, namely that $L D E$ in profit and closing price act in a more than additive (or synergistic) manner in influencing the decision to close an individual trade.

As we employ a large sample size, even a small variation between expected and observed values can generate a significant p-value. To control for the Type-1 error during multiple comparisons we chose the Holm-Bonferroni method (Holm 1979) to provide adjusted p-values. This approach significantly increases the robustness of our proportions' tests. As a further check on the robustness of our results (i.e. to ensure that significant results are not simply arising due to the large sample size involved), we randomly sampled $10 \%$ of the trades (731,457 trades) and repeated the tests on this far smaller sample. This process of random sampling (rudimentary bootstrapping) was repeated ten times with the average values reported in online Appendix 2, Table A2:3. 
Our results suggest that while there are minor differences in the occurrence of rightmost digit profit/closing price combinations in percentage terms, the inferences from the two-sided proportions tests remain the same. This suggests that our significant results are not simply an artefact of the large sample size

\section{Results}

\subsection{Distribution of the Rightmost Digit}

We determined the frequency of trades where the rightmost digits of profit and closing price ranged from 0 to 9, and these are shown in Table 2.

Table 2. Distribution of Rightmost Digit of Profit and Closing Price

\begin{tabular}{cccc}
\hline $\begin{array}{c}\text { Rightmost } \\
\text { Digit }\end{array}$ & $\begin{array}{c}\text { Expected } \\
\text { Frequency }\end{array}$ & $\begin{array}{c}\text { Closing Price } \\
\text { Observed }\end{array}$ & $\begin{array}{c}\text { Profit } \\
\text { Observed }\end{array}$ \\
\hline 0 & 731,457 & 849,882 & $1,561,368$ \\
& $(10 \%)$ & $(11.62 \%)$ & $(21.35 \%)$ \\
1 & 731,457 & 691,509 & 821,214 \\
& $(10 \%)$ & $(9.45 \%)$ & $(11.23 \%)$ \\
2 & 731,457 & 714,251 & 899,110 \\
& $(10 \%)$ & $(9.76 \%)$ & $(12.29 \%)$ \\
3 & 731,457 & 710,305 & 631,272 \\
& $(10 \%)$ & $(9.71 \%)$ & $(8.63 \%)$ \\
4 & 731,457 & 713,334 & 690,524 \\
& $(10 \%)$ & $(9.75 \%)$ & $(9.44 \%)$ \\
5 & 731,457 & 787,727 & 850,132 \\
& $(10 \%)$ & $(10.77 \%)$ & $(11.62 \%)$ \\
6 & 731,457 & 713,369 & 598,615 \\
& $(10 \%)$ & $(9.75 \%)$ & $(8.18 \%)$ \\
7 & 731,457 & 713,576 & 436,336 \\
& $(10 \%)$ & $(9.75 \%)$ & $(5.97 \%)$ \\
8 & 731,457 & 716,756 & 482,366 \\
& $(10 \%)$ & $(9.80 \%)$ & $(6.59 \%)$ \\
9 & 731,457 & 703,861 & 343,633 \\
& $(10 \%)$ & $(9.62 \%)$ & $(4.70 \%)$ \\
\hline
\end{tabular}

Throughout the results section, all two-sided proportions tests were implemented on the basis of the expected and observed frequencies (the percentage figures are simply provided for 
clarity), and each test involved a total population of $N=7,314,570$ observations. Consequently, only the degrees of freedom, $\chi^{2}$ value and $p$-values are reported for each proportions test.

The figures displayed in Table 2 suggest that there may be a LDE associated with realised profit and closing price. A chi ${ }^{2}$ goodness of fit test rejected the null hypothesis of the expected and observed values for realised profit and closing price $\left(\chi^{2}(9)=\right.$ 460,000.00, $\left.p=<0.001 ; \chi^{2}(9)=29,370.00, p=<0.001\right)$.

Two-sided proportions tests utilising expected and observed values from Table 2 suggested that the digits 0 and 5 were overrepresented in both the rightmost digit of realised profit and closing price, highlighting traders' preference for round numbers (0/Profit: $\chi^{2}(1)$ $=35,6230.00, \mathrm{p}=<0.001 ; \quad 5 /$ Profit: $\quad \chi^{2}(1)=9,984.10, \mathrm{p}=<0.001 ; 0 /$ Closing Price: $\chi^{2}(1)$ $=9,943.00, \mathrm{p}=<0.001 ; 5 /$ Closing Price: $\left.\chi^{2}(1)=2,325.70, \mathrm{p}=<0.001\right)$.

In order to explore whether there was a greater LDE associated with closing price or realised profit, we added the frequency of trades whose realised profit ended with a 0 or 5 and we added the frequency of trades whose closing price ended with a 0 or 5 . We found that a round number occurred in the rightmost digit of profit more than in the rightmost digit of closing price $\left(\chi^{2}(1)=204,510.00, p=<0.001\right)$.

The observed frequency of occurrence of each digit 0-9 in profit and closing price alongside their two-sided proportions tests are documented in Appendix 2, Table A2:1 and Table A2:2. Interestingly, the data displayed in Table A2:2 in the online Appendix 2 and the associated proportions tests, indicate that each of the rightmost digits 1-4 and 6-9 of closing price were under-represented. By contrast, the rightmost digits 1 and 2 of realised profit (in addition to the rightmost digits 0 and 5) were over-represented, but the number of trades closed where the rightmost digit of profit was 6 through 9 were substantially lower than that expected (see online Appendix 2, Table A2:1). One explanation for this might be that investors were 
aiming to close their trades on a profit with the rounder digit 0 and overshot their profit target, suggesting that the LDE in profit may be even more pronounced than the raw figures suggest

\subsection{Testing Hypotheses}

Table 3 is a $2 \times 9$ contingency table, detailing the occurrence of all combinations of the digits 0,5 and Other in the rightmost digit of profit and closing price, respectively.

Table 3. Profit and Closing Price Rightmost Digit Combinations Contingency Table

\begin{tabular}{cccccc}
\hline $\begin{array}{c}\text { Rightmost } \\
\text { Digit } \\
\text { Profit / Price }\end{array}$ & $\begin{array}{c}\text { Expected } \\
\text { Frequency }\end{array}$ & $\begin{array}{c}\text { Observed } \\
\text { Frequency }\end{array}$ & $\chi^{2}$ & DF & $\begin{array}{c}\text { Adjusted } \\
\text { p-value }\end{array}$ \\
\hline $0 / 0$ & $73,145.7$ & 183,845 & $48,536.00$ & 1 & $0.000^{* *}$ \\
& $(1 \%)$ & $(2.51 \%)$ & & & \\
$0 / 5$ & $73,145.7$ & 169,769 & $39,082.00$ & 1 & $0.000^{* *}$ \\
& $(1 \%)$ & $(2.32 \%)$ & & & \\
O/Other & 585,180 & $1,207,754$ & $246,380.00$ & 1 & $0.000^{* *}$ \\
& $(8 \%)$ & $(16.51 \%)$ & & & \\
$5 / 0$ & $73,145.7$ & 99,680 & $4,122.30$ & 1 & $0.000^{* *}$ \\
& $(1 \%)$ & $(1.36 \%)$ & & & \\
$5 / 5$ & $73,145.7$ & 94,408 & $2,729.20$ & 1 & $0.000^{* *}$ \\
& $(1 \%)$ & $(1.29 \%)$ & & & \\
$5 /$ Other & 585,180 & 656,044 & $4,420.70$ & 1 & $0.000^{* *}$ \\
& $(8 \%)$ & $(8.97 \%)$ & & & \\
Other/0 & 585,180 & 566,357 & 333.93 & 1 & $0.000^{* *}$ \\
& $(8 \%)$ & $(7.74 \%)$ & & & \\
Other/5 & 585,180 & 523,550 & $3,706.60$ & 1 & $0.000^{* *}$ \\
& $(8 \%)$ & $(7.16 \%)$ & & & \\
Other/Other & $4,681,440$ & $3,813,163$ & $211,650.00$ & 1 & $0.000^{* *}$ \\
& $(64 \%)$ & $(52.13 \%)$ & & & \\
\hline
\end{tabular}

Pearson's goodness of fit test for Table $3, \chi^{2}(8)=1,150,600.00, p=<0.001^{* *}$. Adjusted p-values obtained from the Holm-Bonferroni method. Significant at $99 \%$ confidence interval **

The results presented in Table 3, suggest that several profit/closing price combinations of rightmost digit are overrepresented. However, to test H1, we wanted to ensure that any LDE associated with profit was not simply occurring because it corresponded to a LDE associated with closing price. Consequently, we first focused on two specific combinations of profit/price, namely 0/Other and 5/Other; i.e. where the rightmost digit of profit was a round number but the rightmost digit of price was not. The results of two-sided proportions tests (displayed in 
Table 3) associated with both these combinations are highly significant, suggesting that traders prefer to realise profit with round number rightmost digits (cf. non-round number digits). These results support $\mathrm{H} 1$, that there is a LDE in realised profit. The results also support the findings from a variety of studies across many fields which suggest that individuals use round numbers ( 0 and 5) as cognitive reference points (Pope and Simonsohn 2011; Allen et al, 2016)

To explore the persistence of the LDE in profit and closing price through time, we examined trades made in each of the separate years 2006 to 2013. The results of two-sided proportions tests suggested that the LDE in profit and closing price existed for trades made in each of the years 2006 to 2013 (See Online Appendix 2, Table A2:61 and Table A2:7).

Furthermore, to ensure that our results were robust and not influenced by a handful of frequent traders who exhibited left digit bias (i.e. that the results applied to both frequent and occasional traders), we examined trades made by the top $10 \%$ and $1 \%$ of traders and by the bottom quartile of traders in terms of number of trades (See results in online Appendix 2 Table A2:4). Based on the results of our two-sided proportions tests we found that all these groups (classified by number of trades) exhibited LDE in profit and closing price. The exception was in relation to the rightmost digit 5 for the bottom decile of traders, whereby the observed and expected frequency were similar. One explanation for this is investors with, for example, only one trade, preferred to close their trades on the rounder digit 0 . Overall, these results suggest that the LDE associated with realised profit and closing price is a widespread phenomenon amongst financial traders, mirroring the LDE associated with price found by Kuo et al (2014).

In addition, we checked if the LDE was present if a trade was closed in either profit or loss (see results in Appendix 3, Table 3). The results of our two-sided proportion tests suggest that there is $L D E$ related to both profit and closing price, regardless of whether a trade is closed in profit or loss. In addition, LDE in both metrics is more salient when a trade is closed in loss (Profit: $\quad \chi^{2}(1) \quad=17,796.00, p=<0.001^{* *} ; \quad$ Closing Price: $\quad \chi^{2}(1)$ 
$\left.=4,923.70, p=<0.001^{* *}\right)$. This is a phenomenon which was reported, in relation to closing price, in Fraser-Mackenzie et al (2015), and it appears that this also applies to LDE related to profit. However, this is not the main focus of this paper and will be explored further in a future study.

Lin and Wang (2017) suggest that the LDE in price has a boundary of three digits. They claim individuals are more prone to the LDE in price in numbers up to three digits or less. Beyond three digits, an increased cognitive attention results in a reduced dependence on heuristics, thereby diminishing the LDE.

We found that most realised profits were associated with only one or two digits $(90.24 \%$ of all trades). To explore whether the LDE extended to three digits in realised profit, we conducted a separate analysis (full details provided online in Appendix 1) of all trades with realised profit (cf. loss) of absolute value ranging from $£ 100$ to $£ 999$ (a total of 672,326 trades;19\%). This process would allow us to understand whether a round number middle digit or rightmost digit primarily influenced the decision to close an individual trade. Our findings suggest that the LDE does extend to three figure realised profit, but it is only in the rightmost digit in which we observe this phenomenon. This suggested that the LDE in profit was uniformly present in our entire sample of trades.

In order to test $\mathrm{H} 2$, namely that individuals are more influenced by the LDE associated with the profit than that associated with the closing price, we compared the frequency of occurrence of the profit/closing price combinations of 0/Other and 5/Other with, Other/0 and Other/5, respectively. Each of these combinations has an expected frequency of occurrence of $8 \%$. The relevant frequencies and the results of related proportions tests are shown in Table 4. Table 4. Proportions tests comparing independent LDE in Profit and Closing Price 


\begin{tabular}{|c|c|c|c|c|c|c|}
\hline $\begin{array}{c}\text { Rightmost } \\
\text { Digit } \\
\text { Profit / } \\
\text { Price }\end{array}$ & $\begin{array}{c}\text { Frequency } \\
\text { Observed }\end{array}$ & $\begin{array}{c}\text { Rightmost } \\
\text { Digit } \\
\text { Profit / } \\
\text { Price }\end{array}$ & $\begin{array}{c}\text { Frequency } \\
\text { Observed }\end{array}$ & $\chi^{2}$ & DF & $\begin{array}{c}\text { Adjusted } \\
\text { p-value }\end{array}$ \\
\hline O/Other & $\begin{array}{l}1,207,754 \\
(16.51 \%)\end{array}$ & Other/0 & $\begin{array}{l}566,357 \\
(7.74 \%)\end{array}$ & $263,890.00$ & 1 & $0.000 * *$ \\
\hline 5/Other & $\begin{array}{l}656,044 \\
(8.97 \%)\end{array}$ & Other $/ 5$ & $\begin{array}{l}523,550 \\
(7.16 \%)\end{array}$ & $16,187.00$ & 1 & $0.000 * *$ \\
\hline $\begin{array}{l}\text { Round } \\
\text { No./Other }\end{array}$ & $\begin{array}{l}1,863,798 \\
(25.48 \%)\end{array}$ & $\begin{array}{l}\text { Round } \\
\text { No./Other }\end{array}$ & $\begin{array}{l}1,089,907 \\
(14.90 \%)\end{array}$ & $254,060.00$ & 1 & $0.000 * *$ \\
\hline
\end{tabular}

Adjusted p-values obtained from the Holm-Bonferroni method. Significant at 99\% confidence interval **

The first two rows in Table 3 display results comparing the combinations $0 /$ Other and Other/0 and the combinations 5/Other and Other/5, respectively. The highly significant HolmBonferroni adjusted p-values for both tests lead us to reject the null hypothesis that these combinations represent a similar frequency of trades. Clearly, the combination 0/Other has a greater frequency of occurrence than Other $/ 0(16.51 \% \mathrm{cf}$. $7.74 \%)$, and the combination $5 /$ Other has a greater frequency of occurrence than Other $/ 5$ (8.97\% cf. $7.16 \%)$. The results, therefore, support $\mathrm{H} 2$ and suggest that LDE associated with 0 and 5 in profit has a bigger impact on traders than the LDE associated with these digits in closing price.

We explored the total frequency of trades associated with round numbers in the rightmost digit of profit (i.e. the combinations 0/Other and 5/Other: 25.48\%) with total frequency of trades associated with round numbers in the rightmost digit of closing price (i.e. the combinations Other/0 and Other/5: $14.90 \%$ ). The proportions test results show that these were significantly different values. Consequently, it is clear that whilst the frequency of trades associated with round numbers in the rightmost digits associated with profit is considerably greater than the expected proportion $(25.48 \% \mathrm{cf} .16 \%)$, this is not the case for the frequency of trades associated with round numbers in the rightmost digits associated with closing price $(14.90 \%$ cf. $16 \%)$. Overall, these results suggest that LDE in profit is 1.71 times more influential than the LDE in closing price. Bhattacharya et al (2012) calculated that the LDE in 
stock prices caused negative abnormal returns of $\$ 813$ million per year on the NYSE, suggesting, on the basis of the results reported above that LDE in profit alone could result in substantial negative returns per year for investors.

Based on the results of individual proportions tests documented in Table 3, the frequency of occurrence of profit/price combinations Other/0 and Other $/ 5$ were significantly lower than their expected values. However, the results from Table 3 suggested that the combinations $0 / 0,0 / 5,5 / 0$ and $5 / 5$ were overrepresented.

Our results suggest that the left digit bias or preference for round digits in closing price, though present, is weak unless observed in conjunction with round digit profit. It is possible that the rounded nature of the profit figure is driving the decision to close the trade, while the rounded nature of the price is just coincidental and no different to any other digit. However, such a conclusion would call into question the inferences of several financial market studies (e.g., Bhattarchaya et al, 2012; Kuo et al 2014 and Fraser-Mackenzie et al, 2015) which have found left digit bias in closing price to be present (but a relatively weak effect). Consequently, we feel it more likely that the left digit effect in price is present but is a relatively weak effect compared to the left digit bias associated with profit.

To explore further, as our two sided proportions test suggested the differences in our combinations 0/Other vs. Other/0 were more salient than 5/Other vs.Other/5, we expand the rightmost digit 'Other' category into all relevant digits, 1,2,3,4,6,7,8,9 for the combinations 0/Other vs. Other/0 (See online Appendix 2, Table A2:8 and Table A2:9). The rightmost digit profit/price combination 0/Other has an expected value of 585,180 (8\%) and an observed value of 1,207,754 (16.51\%) and the combination Other/0 has an expected value of 585,180 (8\%) and an observed value of 566,357 (7.74\%) out of 7,314,570 trades. Therefore, each combination of profit and price involving single digits (eight in total) will have an expected frequency of $73,145.70(1 \%)$. 
The proportions tests related to the profit/price rightmost digit combinations 0/Other (displayed in Table A2:8) confirm that every combination (when 'Other' is broken down into each digit 1-4 and 6-9) are over-represented. However, the round digit combinations 0/0 and 0/5 occur more frequently than any other digit combination. These results suggest that traders do place importance on achieving a round number profit and that investors do place some cognitive value in closing prices on round numbers. However, the proportions tests related to the profit/price rightmost digit combinations Other/0 (displayed in Table A2:9) demonstrate that the combinations $3 / 0,6 / 0,7 / 0,8 / 0$ and $9 / 0$ are under-represented and $0 / 0,1 / 0,2 / 0,4 / 0$ and 5/0 are over-represented. In addition, the round digit combination 0/0 occurs far more frequently than any other digit combination and the 5/0 combination occurs more frequently than most other combinations. These results suggest that achieving a round number closing price does have some value to traders. However, the under-representation of some rightmost digit profit/price combinations when profit is not a round number and price is a round number suggests that achieving a round digit price is not paramount to investors. Contrasting these findings with the results displayed in online Appendix 2 Table A2:8, which show that all profit/price rightmost digit combinations 0/Other are over-represented, suggests that achieving a round number rightmost digit profit is more salient to traders than achieving a round number rightmost digit closing price

In order to test $\mathrm{H} 3$, that investors subject to left digit bias will display a preference for rounder numbers in both closing price and profit, we explore whether traders prefer the 'rounder' digit 0 to 5 when closing an individual trade for both profit and closing price. To test this we examine the all-round number combinations (i.e. $0 / 0,0 / 5$ and $5 / 0$ ) and the results are displayed in Table 5. All three were expected to occur with a frequency of $1 \%$, but all were over-represented. These combinations were chosen as they have a common expected frequency (1\%).This simplified comparisons via a proportion test. By comparing the frequency of 
occurrence of these three combinations, we could check whether investors uniformly chose the rounder digit 0 over 5 for both closing price and profit, highlighting universal properties.

Table 5. Comparing LDE on round digits 0 and 5

\begin{tabular}{ccccccc}
\hline $\begin{array}{c}\text { Rightmost } \\
\text { Digit } \\
\text { Profit / } \\
\text { Price }\end{array}$ & $\begin{array}{c}\text { Frequency } \\
\text { Observed }\end{array}$ & $\begin{array}{c}\text { Rightmost } \\
\text { Digit } \\
\text { Profit / } \\
\text { Price }\end{array}$ & $\begin{array}{c}\text { Frequency } \\
\text { Observed }\end{array}$ & $\chi^{2}$ & DF & $\begin{array}{c}\text { Adjusted } \\
\text { p-value }\end{array}$ \\
\hline $0 / 0$ & 183,845 & $0 / 5$ & 169,769 & 574.11 & 1 & $0.000^{* *}$ \\
& $(2.51 \%)$ & $5 / 0$ & $\begin{array}{c}(2.32 \%) \\
99,680 \\
(1.36 \%)\end{array}$ & $25,478.00$ & 1 & $0.000^{* *}$ \\
$0 / 0$ & 183,845 & $5 / 0$ & & & \\
\hline
\end{tabular}

Adjusted p-values obtained from the Holm-Bonferroni method. Significant at 99\% confidence interval **

The first row in Table 5 enables us to explore whether traders prefer rounder numbers in closing price (keeping the rightmost digit in profit constant). The second row enables us to explore whether traders prefer rounder numbers in realised profit (keeping the rightmost digit in closing price constant). The Holm-Bonferroni adjusted p-values $(0.000)$ for both proportions' tests were significant at the $99 \%$ confidence interval, suggesting significant differences in the observed frequencies between the two sets of combinations, $0 / 0-0 / 5$ and $0 / 0-5 / 0$. The observed frequencies in Table 5, suggest that investors preferred to close trades when the rightmost digit in closing price was 0 rather than 5 , as the combination $0 / 0$ occurs more frequently than the combination $0 / 5(2.51 \%$ cf. $2.32 \%)$. Furthermore, the combination $0 / 0$ occurred more frequently than the combination $5 / 0$, suggesting that investors preferred the rightmost digit 0 over 5 in profit $(2.51 \%$ cf. $1.36 \%)$. These results confirmed the findings of Bhattacharya et al. (2012) that there is a 'pecking order' of preference in digits. Furthermore, we found that the pecking order Bhattacharya et al. (2012) observed for price also applies to realised profit, thereby supporting $\mathrm{H} 3$.

We speculated in section 2.4 that if the rightmost digit of both closing price and realised profit were round numbers this might have a synergistic influence on the application of a LDE, and 
this view formed the basis of H4. Our results, reported above, suggest that traders prefer the 'rounder' digit $O$ (cf. 5) for the rightmost digit of both profit and closing price. We therefore, tested $\mathrm{H} 4$ on the more salient rightmost digit 0 , and combined the expected and observed number of observations where the rightmost digit was 5 with those for the Other category, into a new category, Other+. This resulted in the 2 x 4 contingency table shown in Table 6.

Table 6. Comparing the LDE when rightmost digit of profit and closing price are round numbers with situations where this is not the case

\begin{tabular}{cccccc}
\hline $\begin{array}{c}\text { Rightmost } \\
\text { Digit } \\
\text { Profit } / \\
\text { Price }\end{array}$ & $\begin{array}{c}\text { Frequency } \\
\text { Expected }\end{array}$ & $\begin{array}{c}\text { Frequency } \\
\text { Observed }\end{array}$ & $\begin{array}{c}\text { Rightmost } \\
\text { Digit } \\
\text { Profit / Price }\end{array}$ & $\begin{array}{c}\text { Frequency } \\
\text { Expected }\end{array}$ & $\begin{array}{c}\text { Frequency } \\
\text { Observed }\end{array}$ \\
\hline $0 / 0$ & $73,145.70$ & 183,845 & Other $+/ 0$ & $658,311.30$ & 666,037 \\
& $(1 \%)$ & $(2.51 \%)$ & & $(9 \%)$ & $(9.11 \%)$ \\
O/Other+ & $658,311.30$ & $1,377,523$ & Other+/Other+ & $5,924,801.70$ & $5,087,165$ \\
& $(9 \%)$ & $(18.83 \%)$ & & $(81 \%)$ & $(69.55 \%)$ \\
\hline
\end{tabular}

Pearson's goodness of fit test for Table $6, \chi^{2}(3)=1,071,800.00 \mathrm{p}=<0.001^{* *}$. Significant at $99 \%$ confidence interval $* *$

The combinations $0 /$ Other+ and Other $+/ 0$ represent situations where a LDE associated with profit alone or closing price alone might be observed. If the LDEs in profit and closing price were independent, the proportion of observations for the combination $0 / 0$ would simply be the observed proportions of $0 /$ Other + and $O$ ther $+/ 0$ multiplied together (i.e. $0.1883 \times 0.0911=$ 0.0171). However, we observed that $2.51 \%$ of trades were associated with the profit/closing price combination $0 / 0$. The resulting two-sided proportion test suggests that the combined 
effect of a round number in the rightmost digit of both profit and closing price had a greater than additive impact on the overall LDE, thereby supporting $\mathrm{H} 4\left(\chi^{2}(1)=11,270.00, \mathrm{p}=<0.001\right)$.

To formally test the effect size of this observation, we contrasted the frequency of occurrence of trades where the rightmost digit of profit and price were both 0 (i.e. $0 / 0$ ) with the frequency of occurrence of trades involving any other combination of rightmost digits of profit and closing price. As indicated above, if the LDEs in profit and closing price were independent, the proportion of observations for the combination $0 / 0$ would be 0.0171 . Consequently, in Table 7, we include $1.71 \%$ as the expected percentage frequency of trades with a profit/closing price combination of $0 / 0$ and $98.29 \%$ as the expected percentage frequency of trades with any other profit/closing price combination.

Table 7. Contingency table 2 x 2 of Independent and Observed LDE

\begin{tabular}{|c|c|c|c|c|c|}
\hline $\begin{array}{c}\text { Rightmost } \\
\text { Digit } \\
\text { Profit / Price }\end{array}$ & $\begin{array}{c}\text { Expected } \\
\text { Additive } \\
\text { Frequency }\end{array}$ & $\begin{array}{l}\text { Observed } \\
\text { Frequency }\end{array}$ & $\begin{array}{c}\text { Rightmost } \\
\text { Digit } \\
\text { Profit / Price }\end{array}$ & $\begin{array}{c}\text { Expected } \\
\text { Additive } \\
\text { Frequency }\end{array}$ & $\begin{array}{c}\text { Observed } \\
\text { Frequency }\end{array}$ \\
\hline $0 / 0$ & $\begin{array}{l}125,432 \\
(1.71 \%)\end{array}$ & $\begin{array}{l}183,845 \\
(2.51 \%)\end{array}$ & $\begin{array}{c}\text { Any } \\
\text { combination } \\
\text { other than } 0 / 0\end{array}$ & $\begin{array}{l}7,189,138 \\
(98.29 \%)\end{array}$ & $\begin{array}{l}7,130,725 \\
(97.49 \%)\end{array}$ \\
\hline
\end{tabular}

Employing the values shown in Table 7, we determined a Cramer's V value of 0.03 , suggesting that the relative effect size of the synergistic interaction between the LDE for both profit and closing price was relatively small (Cohen, 1990).

However, the absolute effect size, in terms of the total impact of the synergistic impact of a combined LDE related to both profit and closing price on traders' economic well-being was substantial. In particular, the study by Bhattacharya et al (2012) showed the LDE in price alone resulted in negative returns of around $\$ 813$ million per financial year for investors trading in stocks on the NYSE. However, we found the LDE in profit to be 1.71 times more salient than the LDE in closing price. In addition, we found the LDE to be 1.47 times more 
than additive in nature. Thus, despite the fact that Bhattacharya's study was associated with a different trading structure, a different time period, and involved a different set of traders, we speculate that the total negative impact on the returns of investors as a result of the LDE on profit and price, could be staggeringly large.

\section{General Discussion}

Our results confirm traders' preference for realising profit on round numbers. Underpinning this choice is likely to be a cognitive simplification or use of analogue heuristics, whereby individuals mentally map round numbers as reference points (Pope and Simonsohn, 2011). If round numbers are used in this manner, this might result in traders waiting for their individual trades to reach a certain point in terms of profitability rather than reacting rationally and quickly to prevailing market conditions; thereby, adversely affecting their own financial well-being and interfering with market efficiency.

We also found LDE in closing prices, with individuals preferring the rightmost digits 0 and 5 to close trades; thereby, extending the results of Fraser-Mackenzie et al (2015), who found a LDE in prices at which spread trades were opened and closed. This suggests that the LDE remains a persistent bias in various aspects of financial market trading. The persistence of the bias observed in these financial markets is in contrast to several studies in the field of consumer theory, which suggest that the presence of the LDE in the prices of goods and services depends on the context in terms of quality, volume and weight (Kramer 2015; Macé 2012; Thomke and Manzi 2014).

This study found that traders have a greater preference for the digit 0 (cf. 5) as the rightmost digit of profit. Bhattacharya et al (2012) had found a similar pecking order in the digits preferred by investors related to price. However, we find that this preference of 0 over 5 is greater in terms of realised profit than in terms of closing price. Lynn et al (2013) found a 
greater disparity between rounder figures (i.e. 0 cf. 5) in "pay what you feel like" scenarios, and this matches the situation in spread trading.

Our findings suggest that the importance individuals attach to a metric (e.g., profit vs closing price) is crucial in determining the degree of LDE. However, greater freedom in being able to choose the stronger cognitive reference point (0) related to a particular metric, may be one of the factors leading to the LDE being more salient in profit then in closing price.

Our results also suggest that the LDE features more strongly when traders consider their trading profit than when considering the price at which they will close a trade. This finding suggests that traders look primarily at profit while closing an individual trade rather than closing price. Several other studies documenting behavioural biases - namely, the disposition effect and mental accounting - also document profit as the primary metric that impacts the decisions to open and close trades (Barber and Odean 2013;Garvey et al, 2007). Thus, the notion that an individual's foremost aim in trading is to secure a profit is confirmed. We found the salience of the LDE in profit to be 1.7 times more than LDE in closing price, and based on figures calculated by Bhattacharya et al (2012) for investors in the NYSE, this suggests that for these investors alone the LDE related to secured profits could have a substantial negative effect on these investors' returns, which highlights the importance of further research in this field.

In addition, our results suggest that there is a positive synergistic relationship between LDE in profit and closing price, such that the effect is magnified if both these metrics end in round numbers. This can be explained by the Information Integration Theory (Anderson 1971), which suggests that traders in our study cognitively process two pieces of information on round number profit and closing price in a more than additive manner. Taking this into account, we estimated (based on Bhattacharya et al (2012) figures) that the full impact of LDE in profit and closing price may lead to a negative effect on investors returns in the NYSE amounting to about $\$ 3.23$ billion per financial year (assuming that they behave in a similar fashion to the spread 
traders we observe). Thus, the net effect of LDE in all its prevalent forms presents a substantial cognitive barrier to rational decision making and is a barrier to fully efficient markets.

Furthermore, the synergistic nature of the LDE that we observe suggests that its effects may be more significant than previously thought. Dawson et al (2012) showed that despite individuals being aware of the risk hazards of individual phenomena they fail to appreciate fully the combined synergistic risk. Thus, the LDE could manifest itself in other numerical metrics (for example, percentage profit (cf. loss)) used by investors to close an individual trade, thereby magnifying the bias. In addition, the LDE is likely to persist in high-frequency trading algorithms as they are often designed to reflect human heuristics and the programmers themselves may be subject to the LDE. For example, Broussard and Nikiforov (2013) documented programmers' preferences for round digit trading intervals. Therefore, technological advances might increase the negative impact of the LDE. Furthermore, the robustness tests we employ confirm Kuo et al.'s (2014) findings that the LDE persists through time. The large potential impact of this phenomenon which we observe, together with its persistence, suggest the need for prudent intervention, spreading awareness amongst investors.

One of the limitations of our study for making predictions about the impact of the LDE across a range of financial markets, is that our sample primarily involves retail traders. Kuo et al (2014) found that individual or unsophisticated investors are more prone to the LDE. Therefore, it is possible that the impact of the LDE which we observe may be less pronounced for more sophisticated traders. However, the preference for round numbers has been shown to exist in several fields (Jabar and Dia 2014; Allen et al, 2016; Lusher et al, 2018; Pope and Simonsohn 2011). Equally, our study suggests that lay individuals across a range of commercial situations may be subject to the LDE related to profit (not only price, which has been the focus of previous studies). In addition, in our study, the vast majority of the realised profits that we found were subject to the LDE, involved three digits or less. This is similar to 
everyday items purchased by all individuals (as documented by studies into the LDE in consumer theory: Bray and Harris, 2006; Levy et al, 2016). This suggests that our results may have implications for decisions made in a range of consumer settings.

Our results indicate that the LDE may have a considerable impact on trader's actions, and this suggests the need for further study exploring the presence, salience and persistence of the LDE associated with various other numerical metrics (e.g., percentage returns), many of which are readily available to traders, particularly when trading via electronic devices.

\section{Conclusion}

Previous studies exploring the LDE in trading financial assets have only focused on one metric- price. However, profit is the most important numerical metric by which the competence of a trader is measured. This paper is the first to study the presence of the LDE in profit. In the process, we theorise that the salience of the LDE in any metric is dependent on the importance traders ascribe to those metrics. As we anticipated, traders did display a left digit bias related to profit and we showed that this was stronger than that related to the closing price of a trade; suggesting, as we anticipated, that traders assign greater importance to the profit they secure than the final closing price. We also found that traders preferred to close trades when the rightmost digit of the secured profit and/or the closing price was on the 'rounder' digit 0 (cf. 5), suggesting that the former is a more cognitively accessible reference.

Importantly, our results suggest that previous studies have significantly underestimated the impact of the LDE, as these have largely examined LDE in price alone. Our study not only shows that there is an even larger LDE associated with profit, but that there is a positive synergistic interaction between the LDE in realised profit and closing price.

Our study suggests that the LDE could have a substantial impact on investor financial well-being, given the negative consequences for those susceptible to this bias (see Bhattacharya 
et al, 2012). Based on some earlier calculations by Bhattacharya et al, 2012, we estimate that if investors on the NYSE behave in a similar fashion to the traders observed in our study, the combined effect of a LDE on profit and closing price could result in investors in that market alone losing about $\$ 3.23$ billion per financial year. Studies have shown that automated selfcontrol mechanisms such as limit orders used by sophisticated traders to curb other profitoriented biases, are also prone to the LDE (Aşçığlu et al, 2007; Bourghelle and Cellier 2009; Kuo et al, 2014). Consequently, further interventions to aid the traders' decision-making process must be considered in the interests of driving markets towards efficiency.

\section{References}

1. Allen, E.J., P.M. Dechow, D.G. Pope, and G, Wu. 2016. “Reference-dependent preferences: Evidence from marathon runners”. Management Science 63:1657-1672.

2. Anderson, E., and D. Simester. 2003. "Effects of 9 price endings on retail sales: Evidence from field experiments". Quantitative marketing and Economics 1: 93-110.

3. Anderson, N.H.1971."Integration theory and attitude change". Psychological review 78: $171-206$

4. Ansari, S., J. Bell, and H. Okano.2006 "Target costing: uncharted research territory”. Handbooks of management accounting research 2:507-530.

5. Aşçıŏlu, A., C. Comerton-Forde, and T.H. McInish. 2007. "Price clustering on the Tokyo stock exchange”. Financial Review, 42, 289-301.

6. Barber, B., and T. Odean. 2013. "The Behaviour of individual investors", Handbook of Economics of Finance, Elsevier

7. Bhattacharya, U., C.W. Holden, and S. Jacobsen. 2012. "Penny Wise, Dollar Foolish: BuySell Imbalances On and Around Round Numbers" Management Science 58: 413-431 
8. Bourghelle, D., and A. Cellier A., 2009 'Limit order clustering and price barriers on financial markets: Empirical evidence from Euronext”. Working paper, University of Lille, Lille, France.

9. Bray, J.P., and C. Harris.2006. "The Effect of 9-Ending Prices on Retail Sales: A Quantitative UK Based Field Study”. Journal of Marketing Management 22 : 601-617.

10. Broussard, J., and A. Nikiforov. 2013. "Human bias in algorithmic trading".

11. Camilli, G., and L.A. Shepard. 1994. "Methods for identifying biased test items" Sage $4.102-128$

12. Carr, L., and A. Nanni Jr.2009. "Internal Strategy: Organizational Structure". Delivering Results Springer US: 83-101

13. Casarin, R., M. Lazzarin, L. Pelizzon, and D. Sartore. 2005. "Relative benchmark rating and persistence analysis: evidence from Italian equity funds". The European Journal of Finance 11: 297-308.

14. Coulter, K. 2001. "Odd-ending price underestimation: an experimental examination of leftto-right processing effects". Journal of Product and Brand Management 10, 276-292.

15. Chen, G., K. Kim, J.Nofsinger, and O. Rui. 2007. "Trading performance, disposition effect, overconfidence, representativeness bias, and experience of emerging market investors”. Journal of Behavioural Decision Making 20: 425-451.

16. Chou, R.K., and Y.Y. Wang. 2011. "A test of the different implications of the overconfidence and disposition hypotheses". Journal of Banking and finance 35: 2037-2046.

17. Chu, W., M. Im, and H. Jang. 2012. “Overconfidence and emotion regulation failure: How overconfidence leads to the disposition effect in consumer investment Behaviour". Journal of Financial Services Marketing 17:96-116.

18. Cramer, J. 1946. Mathematical Methods of Statistics. Princeton University Press : 282-283

19. Cohen, J. 1990. "Things WE have learned (so far)". American psychologist 45: 1304-1312 
20. Cortez, M.D.C.R., D.A. Paxson, and M.J.D.R. Armada.1999. "Persistence in Portuguese mutual fund performance". The European Journal of Finance 5:.342-365.

21. Dawson, WE.G., J.E. Johnson, and M.A. Luke. 2012. "Do people believe combined hazards can present synergistic risks?”. Risk Analysis 32:801-815.

22. Dhar, R., and N. Zhu.2006. "Up Close and Personal: Investor Sophistication and the Disposition Effect”. Management Science 52: 726-740.

23. Donaldson, R.G. and Kim, H.Y. 1993. "Price barriers in the Dow Jones industrial average". Journal of Financial and Quantitative Analysis, 28: 313-330.

24. Doucouliagos, C. 2005.'Price exhaustion and number preference: time and price confluence in Australian stock prices”. European Journal of Finance 11: 207-221

25. Everaert, J., E.H. Koster, and N. Derakshan.2012. "The combined cognitive bias Hypothesis in depression". Clinical psychology review 32: 413-424.

26. Fischbacher, U., G. Hoffmann, and S. Schudy.2017. "The causal effect of stop-loss and take-gain orders on the disposition effect" . The Review of Financial Studies 30: 2110-2129.

27. Fraser-Mackenzie, P., M. Sung, and J.E.V. Johnson.2015. "The prospect of a perfect ending: Loss aversion and the round-number bias". Organizational Behaviour and Human Decision Processes 131: 67-80.

28. Garvey, R., A. Murphy, and F. Wu. 2007. “Do Losses Linger?”. The Journal of Portfolio Management 33: 75-83.

29. Hirsch, C.R., D.M. Clark, A. Mathews.2006. "Imagery and interpretations in social phobia: Support for the combined cognitive biases Hypothesis”. Behaviour Therapy 37: 223236.

30. Holm, S.1979.” A simple sequentially rejective multiple test procedure”. Scandinavian Journal of Statistics. 6: 65-70

31. Hussain, N.Z., and H. Jones.2016. "Britain clamps down on spread betting industry", Reuters, $6^{\text {th }}$ December, Accessed, $30^{\text {th }}$ May, 2018; 
https://uk.reuters.com/article/uk-britain-regulation-derivatives-dUKKBN13V0UA

32. Jaber, M., and M. Dia .2014. "Foreign Currency Pricing and Left-Digit Pricing". Society for Marketing Advances Proceedings, 286-287.

33. Johnson, E., N. Johnson, and D. Shanthikumar.2007. "Round Numbers and Security Returns”. Working Paper 678, Harvard Business School.

34. Kahneman, D., and A. Tversky.1979. "Prospect theory: An analysis of decision under risk". Econometrica 47: 263-292

35. Kateri, M. 2014. Contingency table analysis. Springer New York: 1-15

36. Kim, J., H.J. Yoon, and S.Y. Lee.2010. "Integrating advertising and publicity". Journal of Advertising 39:97-114.

37. Kramer, A.2015. "Robustness of Price Perception: How Strong are Anchoring, Left-Digitand Framing-Effects when Promoting Sales Offers?". Business and Management Studies 1: $35-43$

38. Kuo, W., T. Lin, and J. Zhao.2014. "Cognitive Limitation and Investment Performance: Evidence from Limit Order Clustering”. Review of Financial Studies 28: 838-875.

39. Lean, H.H., W.R. Ang, and R. Smyth. 2015. "Performance and performance persistence of socially responsible investment funds in Europe and North America". The North American Journal of Economics and Finance 34: 254-266.

40. Levy, D., D. Lee, H. Chen, R.J. Kauffman, and Bergen, M. 2011. "Price points and price rigidity". Review of Economics and Statistics 93: 1417-1431.

41. Levy, D., A. Snir, A. Gotler, H. Chen.2016. "Not all price endings are created equal: price points and asymmetric price rigidity". Unpublished Manuscript

42. Lim, S. 2004. "Do Investors Integrate Losses and Segregate Gains? Mental Accounting and Investor Trading Decisions", EFA 2003 Annual Conference Paper No. 87 
43. Lin, C.H., and J.W. Wang.2017." Distortion of price discount perceptions through the leftdigit effect". Marketing Letters 28: 99-112.

44. Lin, C.H., and J.W. Wang.2011." Effect of font size and appearance in left-digit price cognition”. African journal of business management 5: 9541-9547.

45. Linnainmaa, J.T. 2010. "Do limit orders alter inferences about investor performance and behaviour?". The Journal of Finance 65: 1473-1506.

46. Lusher, L., C. He, and S. Fick.2018. "Are professional basketball players referencedependent?". Applied Economics 50: 3937-3948.

47. Lynn, M., S.M. Flynn, and C. Helion.2013. "Do consumers prefer round prices? Evidence from pay-what-you-want decisions and self-pumped gasoline purchases". Journal of Economic Psychology 36: 96-102.

48. Macé, S.2012."The impact and determinants of nine-ending pricing in grocery retailing”. Journal of Retailing 88: 115-130.

49. Manning, K., and D. Sprott.2009.'Price endings, left-digit effects, and choice”. Journal of Consumer Research 36: 328-335.

50. Odean, T.1998. “Are Investors Reluctant to Realize Their Losses?”. The Journal of Finance 53: $1775-1798$

51. Pope, D., and U. Simonsohn.2011."Round numbers as goals: Evidence from baseball, SAT takers, and the lab". Psychological science 22: 71-79.

52. Richards, D.W., J. Rutterford, D. Kodwani, M. Fenton-O'Creevy. 2017. "Stock market investors' use of stop losses and the disposition effect". The European Journal of Finance 23:130-152.

53. Rodrigue, C.S., and A. Biswas.2004. "Brand alliance dependency and exclusivity: an empirical investigation”. Journal of Product and Brand Management 13: 477-487. 
54. Schindler, R., and T. Kibarian.2001. "Image communicated by the use of 99 endings in advertised prices". Journal of Advertising 30:95-99.

55. Shefrin, H., and M. Statman.1985. "The Disposition to Sell Winners Too Early and Ride Losers Too Long: Theory and Evidence”. Journal of Finance 40: 777-790

56. Sonnemans, J.2006. "Price clustering and natural resistance points in the Dutch stock market: A natural experiment”. European Economic Review 50:1937-1950.

57. Sopranzetti, B., and V. Datar.2002. "Price clustering in foreign exchange spot markets". Journal of Financial Markets 5:411-417.

58. Stiving, M., and R. Winer.1997. "An empirical analysis of price endings with scanner data”. Journal of Consumer Research 24: 57-67.

59. Tarpey, T.2000. "A note on the prediction sum of squares statistic for restricted least squares". The American Statistician 54:116-118.

60. Thaler, R.1985. "Mental accounting and consumer choice". Marketing science 4: 199-214.

61. Thaler, R., and E. Johnson.1990. "Gambling with the house money and trying to break even: The effects of prior outcomes on risky choice". Management science 36: 643-660.

62. Thomas, M., and V. Morwitz.2004. "Effects of Framing on Magnitude Perceptions of Prices". Advances in Consumer Research 31: 454-456.

63. Thomas, M., and V. Morwitz.2005. "Penny Wise and Pound Foolish: The Left-Digit Effect in Price Cognition". Journal of Consumer Research 1: 54-64.

64. Thomke, S., and J. Manzi.2014."The Discipline of Business Experimentation", Harvard Business Review 92: 71-79. 